\title{
Poisson Geometry of the Analog of the Miura Maps and Bäcklund-Darboux Transformations for Equations of Toda Type and Periodic Toda Flows
}

\author{
Percy Deift ${ }^{1}$ and Luen-Chau $\mathbf{L i}^{2}$ \\ 1 Courant Institute, New York, NY 10012, USA \\ 2 Dept. of Mathematics, 218 Mc Allister Building, Pennsylvania State University, \\ University Park, PA 16802, USA
}

Received April 19, 1991

\begin{abstract}
We show that the analog of the Miura maps and Bäcklund-Darboux transformations for a general class of equations of Toda type and for a generalized class of periodic Toda flows are isomorphisms of Poisson Lie groups.
\end{abstract}

\section{Introduction}

In the study of the Korteweg-de Vries (KdV) equation, it is well-known that an important role is played by the Miura maps which relate $\mathrm{KdV}$ to an associated modified equation $(\mathrm{mKdV})[\mathrm{M}]$. On the other hand, Bäcklund-Darboux transformations have been used successfully to construct soliton solutions (see, e.g. [WE, D]). If $L=-D^{2}+q$ and $L=A^{*} A$ denotes the factorization of $L$ into first order operators, where $A=D-v$, then the starting point in [D], for example, is the observation that the Bäcklund-Darboux transformation for $\mathrm{KdV}$ corresponds to a reordering of factors in $L$, i.e. $L=A^{*} A \mapsto \widetilde{L}=A A^{*}$. As has been noted by Adler [A], there is also a parallel theory for the periodic Toda lattice. In this case, the analog of the Miura maps takes the $2 n$-periodic Kac-van Moerbeke lattice [KvM] into the $n$-periodic Toda lattice, and again, the Bäcklund-Darboux transformation corresponds to a factorization and reordering of the factors. The Gelfand-Dickey hierarchy [GD1] generalizes the KdV hierarchy to $n^{\text {th }}$ order operators, and in this context, the generalized Miura maps were introduced and analyzed by Kupershmidt and Wilson [KW]. In [KW] it was shown, for the first time, that the Miura maps, and their $n^{\text {th }}$ order generalizations, were canonical with respect to appropriate Poisson structures. This involves a so-called Adler-Gelfand-Dickey second structure [GD2], which is a forerunner of a general construction on associative algebras for skew-symmetric $r$-matrices [STS1]. By contrast, nothing is known about the Hamiltonian character of the Miura maps for the case of the periodic Toda lattice. From a different direction, in the study of the interpolation of discrete algorithms in numerical linear algebra by continuous flows, it was observed that if an $n \times n$ real matrix $M$ solves the so-called SVD flow, then both 
$M M^{T}$ and $M^{T} M$ are solutions of the Toda equation [C, DDLT]. Again, the question of whether the maps $M \mapsto M M^{T}, M^{T} M$ are Poisson maps is open.

The purpose of this paper is two-fold. On the one hand, we would like to understand the Hamiltonian nature of the analog of the Miura maps for a general class of systems of Toda type, and also for a generalized class of periodic Toda flows (see Sects. 3 and 4). Our approach to this question builds on recent progress in [LP]. By working on the group level, our second purpose is to understand the extended Sklyanin bracket in [LP], particularly with regard to its relation with Poisson Lie groups. Let us recall [STS2] that a Lie group $G$ equipped with the Sklyanin bracket is a Poisson Lie group, i.e., the multiplication map is a Poisson map from $G \times G$ (equipped with the product structure) into $G$. For a Poisson Lie group, the underlying Poisson structure is uniquely determined by its linearization at the identity. Also, it follows from a theorem of Manin [Drin] that there exists a dual Poisson Lie group. With the modified structure in [LP], $G$ is no longer a Poisson Lie group and the above properties are unfortunately lost. However, as we will show, the image of the analog of the Miura map(s) is a Poisson submanifold of the Lie group equipped with the modified structure. Furthermore, this Poisson submanifold (with the induced structure) can be endowed with two distinct multiplication maps which turn it into isomorphic Poisson Lie groups, the isomorphism being supplied by the Bäcklund-Darboux transformation! In particular, our arguments also show that the restriction of the Bäcklund-Darboux transformation to the classical (periodic and non-periodic) Toda lattice is also a Poisson map.

The paper is organized as follows. In Sect. 2, for the convenience of the reader, we assemble some of the basic facts about Poisson structures on Lie groups. The main results are in Sects. 3 and 4. Section 3 is concerned with systems of Toda type on real, semisimple Lie groups. We introduce these systems and the associated modified equations. Then we study the Poisson geometry of the analog of the Miura maps and the Bäcklund-Darboux transformation. As an application of our results, we establish the complete integrability of the SVD flow [C, DDLT] on the upper triangular group. In Sect.4, we do the same for the generalized periodic Toda flows, where the dynamics now takes place on appropriate infinitedimensional loop groups. We close the paper by considering the classical periodic Toda lattice. Here, the maps which take the $2 n$-periodic Kac-van Moerbeke lattice to the $n$-periodic Toda lattice are shown to be Poisson maps.

This paper was motivated by a question which arose in [DDLT]. As noted above, the map $M \mapsto M^{T} M$ (or $M M^{T}$ ) takes the SVD flow into the Toda flow. In [DDLT], the SVD flow was shown to be Hamiltonian with respect to a Sklyanin structure. On the other hand, the Toda flow is Hamiltonian with respect to the LiePoisson structure on symmetric matrices, regarded as the dual Lie algebra of the upper triangular group [S]. The map $M \mapsto M^{T} M$ (or $M M^{T}$ ), however, is clearly not canonical with respect to these two structures, and as noted above, the Hamiltonian nature of the map(s) is not clear. In Sect. 3, however, we show that $M \mapsto M^{T} M$ (or $M M^{T}$ ) is indeed canonical, if the domain retains the Sklyanin structure, but the range, consisting of positive definite matrices, is equipped with a modified structure in [LP].

Finally, we note that, at the general level of semisimple Lie algebras, the underlying thrust of the results that follow is an interplay of the Iwasawa and Cartan decompositions of a Lie algebra, moderated by the analog of the Miura map. However, our understanding of the interplay is not yet complete. 


\section{Poisson Structures on Lie Groups}

We review in this section some of the basic facts about Poisson structures on Lie groups which will be used in the sequel. For systematic treatments and proofs, we refer the reader to [STS2, LP].

Definition 2.1. (a) A Lie group $G$ equipped with a Poisson structure is called a Poisson Lie group if group multiplication is a Poisson map from $G \times G$ (equipped with the product structure) into $G$.

(b) Let $G$ be a Poisson Lie group and $H$ a Lie subgroup of $G$. We say $H$ is a Poisson Lie subgroup of $G$ if $H$ is a Poisson submanifold of $G$ and forms a Poisson Lie group with respect to the induced structure.

A Poisson Lie group has the special property that the underlying Poisson structure is uniquely determined by its linearization at the identity. An important class of Poisson Lie groups is associated with the so-called classical $r$-matrices.

Definition 2.2. Let $g$ be a Lie algebra. A linear operator $R \in E n d g$ is called a classical $r$-matrix if the formula

$$
[X, Y]_{R}=\frac{1}{2}([R X, Y]+[X, R Y]), \quad X, Y \in \mathfrak{g}
$$

defines a Lie bracket. We shall denote by $\mathfrak{g}_{R}$ the vector space $g$ when equipped with $[,]_{R}$.

From the point of view of integrable systems theory, operators $R$ which satisfy the modified Yang-Baxter equation

$$
[R X, R Y]-2 R[X, Y]_{R}=-[X, Y], \quad X, Y \in \mathfrak{g},
$$

which is sufficient for $R$ to be a classical $r$-matrix [STS1], are of particular interest.

Now, consider a Lie group $G$ whose Lie algebra $\mathfrak{g}$ is equipped with a nondegeneratead-invariant pairing $(\cdot, \cdot)$. For $\varphi \in C^{\infty}(G)$, we define the left and right gradients $D^{\prime} \varphi, D \bar{\varphi}: G \rightarrow \mathfrak{g}$ by

We have

$$
\left(D^{\prime} \varphi(g), X\right)=\left.\frac{d}{d t}\right|_{t=0} \varphi\left(g e^{t X}\right), \quad(D \varphi(g), X)=\left.\frac{d}{d t}\right|_{t=0} \varphi\left(e^{t X} g\right) .
$$

Theorem 2.3 [STS2]. Let $R \in$ Endg be a skew-symmetric solution of (mYB), then

$$
\{\varphi, \psi\}_{\text {Skly. }}=\frac{1}{2}\left(R\left(D^{\prime} \varphi\right), D^{\prime} \psi\right)-\frac{1}{2}(R(D \varphi), D \psi), \quad \varphi, \psi \in C^{\infty}(G)
$$

defines a Poisson structure on $G$, known as the Sklyanin bracket. Moreover, $\left(G,\{,\}_{\text {skly. }}\right)$ is a Poisson Lie group.

The Sklyanin structure admits an extension for a class of $r$-matrices which includes the skew-symmetric ones. In [LP], the basic assumption on $R \in$ End $g$ is:

$$
R \text { and } A=\frac{1}{2}\left(R-R^{*}\right) \text { are solutions of (mYB). }
$$

Theorem $2.4[\mathrm{LP}]$. Suppose $R$ satisfy hypothesis $(\mathrm{H})$ above and $S=\frac{1}{2}\left(R+R^{*}\right)$, then the formula

$$
\begin{array}{r}
\{\varphi, \psi\}=\frac{1}{2}\left(A\left(D^{\prime} \varphi\right), D^{\prime} \psi\right)-\frac{1}{2}(A(D \varphi), D \psi)+\frac{1}{2}\left(S(D \varphi), D^{\prime} \psi\right) \\
-\frac{1}{2}\left(S\left(D^{\prime} \varphi\right), D \psi\right), \quad \varphi, \psi \in C^{\infty}(G)
\end{array}
$$

defines a Poisson structure on $G$. 
In contrast to the Sklyanin bracket, the group $G$ equipped with the modified structure in Theorem 2.4 is not a Poisson Lie group. Therefore, the Hamiltonian operator $\eta: G \rightarrow$ End $g$ defined by $\{\varphi, \psi\}(g)=(\eta(g) D \varphi(g), D \psi(g))$ is not a 1 cocycle of $G$ for the Ad-action.

\section{Analog of Miura Maps and Bäcklund-Darboux Transformations for Equations of Toda Type}

We introduce Lax equations of Toda type and the associated modified equations on real, semisimple Lie groups. Then we describe the Poisson geometry of the analog of the Miura maps and the Bäcklund-Darboux transformations. As an application, we prove the complete integrability of the SVD flow [C] on the upper triangular group.

Let $\mathfrak{g}$ be a semi-simple Lie algebra over $\mathbb{C}$, and $\langle\cdot, \cdot\rangle$ the associated CartanKilling form. We fix a Cartan subalgebra $\mathfrak{h}$ of $\mathfrak{g}$, and let $\Delta$ be the root system for the pair $(\mathfrak{g}, \mathfrak{h})$. Then we have the root space decomposition

$$
\mathfrak{g}=\mathfrak{h}+\sum_{\alpha \in \Delta} \mathfrak{g}_{\alpha} .
$$

Suppose $\left\{H_{1}, \ldots, H_{l}\right\}$ is a basis of $\mathfrak{h}_{\mathbb{R}}$, and $\Delta^{+} \subset \Delta$ is a positive system. According to [V], there exists an involution $\tau \in \operatorname{Aut}(\mathfrak{g})$ such that $\left.\tau\right|_{\mathfrak{h}}=-1$; and also one can select, for each $\alpha \in \Delta^{+}$, a $Z_{\alpha} \in \mathfrak{g}_{\alpha}$ such that $\left\langle Z_{\alpha}, \tau\left(Z_{\alpha}\right)\right\rangle=-1$. Set $Z_{-\alpha}=-\tau\left(Z_{\alpha}\right)$, $\alpha \in \Delta^{+}$. Then the $H_{i}(1 \leqq i \leqq l)$ and the $Z_{\alpha}(\alpha \in \Delta)$ form a Weyl basis [V]. The real, semisimple Lie algebra

$$
\mathfrak{g}_{0}=\mathfrak{h}_{\mathbb{R}}+\sum_{\alpha \in \Delta} \mathbb{R} \cdot Z_{\alpha}
$$

is the real normal form of $\mathfrak{g}$, and has subalgebras

$$
\mathfrak{l}=\sum_{\alpha \in \Delta^{+}} \mathbb{R} \cdot\left(Z_{\alpha}-Z_{-\alpha}\right), \quad \mathfrak{u}=\mathfrak{h}_{\mathbb{R}}+\sum_{\alpha \in \Delta^{+}} \mathbb{R} \cdot Z_{\alpha} .
$$

We have the Iwasawa decomposition $\mathfrak{g}_{0}=\mathfrak{l} \oplus \mathfrak{u}$ and the Cartan decomposition $\mathfrak{g}_{0}=\mathfrak{f} \oplus \mathfrak{p}$ into +1 and -1 eigenspaces of $\tau$. Let $G_{0}$ be a connected Lie group with Lie algebra $g_{0}$. Then $G_{0}=K \cdot U$, where $K$ and $U$ are analytic subgroups of $G_{0}$ with Lie algebras $\mathfrak{f}$ and $\mathfrak{u}$ respectively. On the other hand, the map $\mathfrak{p} \times K$ $\rightarrow G_{0}:(X, k) \mapsto(\exp X) k$ is a diffeomorphism and we denote $\exp (\mathfrak{p})$ by $P$. Now, let $\Pi_{\mathfrak{t}}, \Pi_{\mathfrak{u}}$ denote the projection operators relative to the Iwasawa decomposition of $\mathfrak{g}_{0}$. We define

$$
R=\Pi_{\mathfrak{f}}-\Pi_{\mathfrak{u}}, \quad A=\frac{1}{2}\left(R-R^{*}\right) \text { and } S=\frac{1}{2}\left(R+R^{*}\right) .
$$

Proposition 3.1. $R$ is a solution of (mYB) which satisfies hypothesis $(\mathrm{H})$.

Proof. Since $\mathfrak{f}$ and $\mathfrak{u}$ are subalgebras of $\mathfrak{g}_{0}$, it follows from [STS1] that $R$ is a solution of (mYB). For $X \in g_{0}$, let $X_{0}, X_{+}$and $X$ - denote the components of $X$ in $\mathfrak{h}_{\mathbb{R}}, \operatorname{span}\left\{Z_{\alpha}\right\}_{\alpha \in \Delta^{+}}$and $\operatorname{span}\left\{Z_{-\alpha}\right\}_{\alpha \in \Delta^{+}}$respectively. Then $\Pi_{\mathfrak{f}} X=X_{-}+\tau X_{-}, \Pi_{\mathfrak{u}} X$ $=X_{0}-\tau X_{-}+X_{+}$. By a straightforward computation, we find that $\Pi_{\mathrm{f}}^{*} X=X_{+}$ $+\tau X_{-}$and $\Pi_{\mathfrak{u}}^{*} X=X_{0}+X_{-}-\tau X_{-}$. Therefore, $A X=X_{-}-X_{+}$and $A$ can be easily shown to be a solution of (mYB).

In what follows, we denote the lift of the Cartan involution $\tau$ on $g$ to the group level also by the same symbol. 
Definition 3.2. (a) A Lax equation of Toda type on $G_{0}$ is an equation of the form

$$
\dot{g}=\frac{1}{2} T_{e} R_{g}(R(D \varphi(g)))-\frac{1}{2} T_{e} R_{g}(R(D \varphi(g))),
$$

where $\varphi \in C^{\infty}\left(G_{0}\right)$ is a central function, i.e. $\varphi\left(h g h^{-1}\right)=\varphi(g), g, h \in G_{0}$.

(b) The modified Toda equation on $G_{0}$ associated with a central function $\varphi$ satisfying $\varphi\left(\tau\left(h^{-1}\right)\right)=\varphi(h), h \in G_{0}$, is the equation

$$
\dot{g}=\frac{1}{2} T_{e} R_{g}\left(A\left(D h_{\varphi}(g)\right)\right)-\frac{1}{2} T_{e} L_{g}\left(A\left(D^{\prime} h_{\varphi}(g)\right)\right),
$$

where $h_{\varphi}(g)=\varphi\left(\tau\left(g^{-1}\right) g\right)$.

Remark. Observe that on matrix groups, (3.5) is an equation in Lax pair form. A model for (3.6) is the SVD flow in [C, DDLT].

Both Eqs. (3.5) and (3.6) are Hamiltonian systems on $G_{0}$. Indeed we have [LP]

Proposition 3.3. (a) Equation (3.5) is the Hamilton's equation generated by $\varphi$ in the modified Poisson structure

$$
\begin{aligned}
\left\{\varphi_{1}, \varphi_{2}\right\}=\frac{1}{2}\left\langle A\left(D^{\prime} \varphi_{1}\right), D^{\prime} \varphi_{2}\right\rangle & -\frac{1}{2}\left\langle A\left(D \varphi_{1}\right), D \varphi_{2}\right\rangle \\
& +\frac{1}{2}\left\langle S\left(D \varphi_{1}\right), D^{\prime} \varphi_{2}\right\rangle \\
& -\frac{1}{2}\left\langle S\left(D^{\prime} \varphi_{1}\right), D \varphi_{2}\right\rangle, \varphi_{1}, \varphi_{2} \in C^{\infty}\left(G_{0}\right) .
\end{aligned}
$$

(b) Equation (3.6) is generated by the Hamiltonian $h_{\varphi}$ in the Sklyanin structure

$$
\begin{gathered}
\left\{\varphi_{1}, \varphi_{2}\right\}_{\text {Skly. }}=\frac{1}{2}\left\langle A\left(D^{\prime} \varphi_{1}\right), D^{\prime} \varphi_{2}\right\rangle-\frac{1}{2}\left\langle A\left(D \varphi_{1}\right), D \varphi_{2}\right\rangle, \\
\varphi_{1}, \varphi_{2} \in C^{\infty}\left(G_{0}\right) .
\end{gathered}
$$
as

Using the fact that $\varphi$ is a central function, it is clear that we can rewrite Eq. (3.5)

$$
\dot{g}=T_{e} R_{g}\left(\Pi_{\mathfrak{t}} D \varphi(g)\right)-T_{e} L_{g}\left(\Pi_{\mathfrak{f}} D \varphi(g)\right) .
$$

Using formula (3.10a) and (3.10b) below, together with the fact that $\varphi$ is central, we find $D h_{\varphi}, D^{\prime} h_{\varphi} \in \mathfrak{p}$. As $A X=\Pi_{\mathfrak{f}} X$ for all $X \in \mathfrak{p}$, we conclude that Eq. (3.6) is equivalent to

$$
\dot{g}=\frac{1}{2} T_{e} R_{g}\left(\Pi_{\mathfrak{f}} D h_{\varphi}(g)\right)-\frac{1}{2} T_{e} L_{g}\left(\Pi_{\mathfrak{t}} D^{\prime} h_{\varphi}(g)\right) .
$$

At this juncture, we introduce maps $\alpha, \beta: G_{0} \rightarrow P$,

$$
\alpha(g)=\tau\left(g^{-1}\right) g, \quad \beta(g)=g \tau\left(g^{-1}\right), \quad g \in G_{0} .
$$

Remark. If $G=S L(n, \mathbb{C})$, then for the standard choice of the Cartan subalgebra (see Remark 3.10 below), $G_{0}=S L(n, \mathbb{R})$ and $\tau(g)=\left(g^{-1}\right)^{T}, g \in G_{0}$. Therefore, $\alpha$ and $\beta$ reduce to the maps discussed in the introduction.

The reason we call Eq. (3.6) a modified Toda equation is due to the following result.

Proposition 3.4. If $g$ satisfies Eq. (3.8), then $\alpha(g)$ and $\beta(g)$ satisfy the Lax equation (3.7).

Proof. We give the verification of the assertion for $\alpha(g)$. From the equation satisfied by $g$, it is easy to check that

$$
(\alpha(g))^{\cdot}=\frac{1}{2} T_{e} R_{\alpha(g)}\left(\Pi_{\mathfrak{f}} D^{\prime} h_{\varphi}(g)\right)-\frac{1}{2} T_{e} L_{\alpha(g)}\left(\Pi_{\mathrm{f}} D^{\prime} h_{\varphi}(g)\right) .
$$


As $\varphi$ is central and is invariant under the composition of the inversion map and $\tau$, we find from formula (3.10b) below that

$$
D^{\prime} h_{\varphi}(g)=D \varphi(\alpha(g))-\tau(D \varphi(\alpha(g)))=2 D \varphi(\alpha(g)) .
$$

Hence the assertion follows.

As a first step towards establishing the Hamiltonian nature of the maps $\alpha$ and $\beta$ introduced above, we first prove

Proposition 3.5. $P$ is a Poisson submanifold of $\left(G_{0},\{\},\right)$.

Proof. The Hamiltonian vector field generated by $\psi \in C^{\infty}\left(G_{0}\right)$ in the Poisson structure $\{$,$\} of Proposition 3.3$ is given by

$$
\begin{aligned}
X_{\varphi}(g)=T_{e} R_{g} \Pi_{\mathfrak{f}}\left(D^{\prime} \psi(g)+D \psi(g)\right) & -T_{e} L_{g} \Pi_{\mathfrak{f}}\left(D^{\prime} \psi(g)+D \psi(g)\right) \\
& +T_{e} R_{g} \Pi_{\mathfrak{u}}^{*}\left(D \psi(g)-D^{\prime} \psi(g)\right) \\
& +T_{e} L_{g} \Pi_{\mathfrak{u}}^{*}\left(D \psi(g)-D^{\prime} \psi(g)\right) .
\end{aligned}
$$

Now, take a point $p=\exp (s) \in P$; we must show $X_{\psi}(p) \in T_{p} P$. Consider the first two terms in the above expression for $X_{\psi}$ evaluated at $p$. This is equal to

$$
T_{e} R_{\exp (s)} \Pi_{\mathfrak{f}}\left(D^{\prime} \psi+D \psi\right)-T_{e} L_{\exp (s)} \Pi_{\mathfrak{f}}\left(D^{\prime} \psi+D \psi\right)=\left.\frac{d}{d t}\right|_{t=0} \exp \left(\operatorname{Ad}_{\left.e^{t \Pi \Pi_{\mathfrak{t}}\left(D^{\prime} \psi+D \psi\right)} s\right)}\right.
$$

which lies in $T_{p} P$ as $\operatorname{Ad}_{e^{t \Pi_{\mathfrak{t}}\left(D^{\prime} \psi+D \psi\right)}} S$ is in $\mathfrak{p}$. For the other two terms, the evaluation at $p$ gives

$$
\begin{aligned}
& T_{e} R_{\exp (s)} \Pi_{\mathfrak{u}}^{*}\left(D \psi-D^{\prime} \psi\right)+T_{e} L_{\exp (s)} \Pi_{\mathfrak{u}}^{*}\left(D \psi-D^{\prime} \psi\right) \\
& \quad=\left.\frac{d}{d t}\right|_{t=0} \exp \left(t \Pi_{\mathfrak{u}}^{*}\left(D \psi-D^{\prime} \psi\right)\right) \exp (s) \exp \left(t \Pi_{\mathfrak{u}}^{*}\left(D \psi-D^{\prime} \psi\right)\right)
\end{aligned}
$$

But the image of $\Pi_{\mathfrak{u}}^{*}$ lies in $\mathfrak{p}$, and so

$$
\exp \left(t \Pi_{\mathfrak{u}}^{*}\left(D \psi-D^{\prime} \psi\right)\right) \exp (s) \exp \left(t \Pi_{\mathfrak{u}}^{*}\left(D \psi-D^{\prime} \psi\right)\right)=\tau\left(c(t)^{-1}\right) c(t) \in P,
$$

where $c(t)=\exp \left(\frac{1}{2} s\right) \exp \left(t \Pi_{\mathfrak{u}}^{*}\left(D \psi-D^{\prime} \psi\right)\right)$. Consequently, the evaluation of the last two terms at $p$ is also in $T_{p} P$.

The induced Poisson structure on $P$ will be denoted by $\{,\}_{P}$.

Theorem 3.6. The maps $\alpha, \beta:\left(G_{0},\{,\}_{\text {Skly. }}\right) \rightarrow\left(P,\{,\}_{P}\right)$ are Poisson maps.

The proof of this result is based on the following properties of the operators $A$ and $S$.

Lemma 3.7. For all $X \in \mathfrak{g}_{0}$,

(a) $\tau(A X)=-A(\tau X)$

(b) $A(X)+S(\tau X)=X$.

Proof. For $X \in \mathrm{g}_{0}$, let $X_{0}, X_{+}$, and $X_{-}$be as in the proof of Proposition 3.1. Then (a) follows on noting that $\tau\left(X_{\mp}\right)=(\tau X)_{ \pm}$. On the other hand, it is easy to verify that $S(\tau X)=X_{0}+2 X_{+}$. As $A(X)=X_{-}-X_{+}$, (b) follows.

Proof of Theorem 3.6. We shall prove the assertion for $\alpha$. Let $\bar{\varphi}, \bar{\psi} \in C^{\infty}(P)$ and extend them to functions $\varphi, \psi$ on $G_{0}$. We have to show $\{\varphi \circ \alpha, \psi \circ \alpha\}_{\text {Skly. }}=\{\varphi, \psi\} \circ \alpha$. 
By a direct computation, we find

$$
\begin{gathered}
D(\varphi \circ \alpha)(g)=D\left(\varphi \circ L_{\tau\left(g^{-1}\right)}\right)(g)-\tau\left(D\left(\varphi \circ L_{\tau\left(g^{-1}\right)}\right)(g)\right), \\
D^{\prime}(\varphi \circ \alpha)(g)=D^{\prime} \varphi(\alpha(g))-\tau(D \varphi(\alpha(g))) .
\end{gathered}
$$

In particular, as noted earlier, (3.10a) implies $D(\varphi \circ \alpha)(g) \in \mathfrak{p}$ and so $A(D(\varphi \circ \alpha)(g)) \in \mathfrak{f}$. As $\langle\mathfrak{f}, \mathfrak{p}\rangle=0$, it follows that

$$
\begin{aligned}
& 2\{\varphi \circ \alpha, \psi \circ \alpha\}_{\text {Skly. }}(g) \\
& \quad=\left\langle A\left(D^{\prime}(\varphi \circ \alpha)(g)\right), D^{\prime}(\psi \circ \alpha)(g)\right\rangle \\
& \quad=\left\langle A\left(D^{\prime} \varphi(\alpha(g))-\tau(D \varphi(g)), D^{\prime} \psi(\alpha(g))-\tau(D \psi(\alpha(g)))\right\rangle .\right.
\end{aligned}
$$

To simplify notation, let $X=D \varphi(\alpha(g)), \quad Y=D \psi(\alpha(g)), \quad X^{\prime}=D^{\prime} \varphi(\alpha(g)) \quad$ and $Y^{\prime}=D^{\prime} \psi(\alpha(g))$. Now, invoke Lemma 3.7, we obtain

$$
\begin{aligned}
& 2\{\varphi \circ \alpha, \psi \circ \alpha\}_{\text {Skly. }}(g) \\
& \quad=\left\langle A\left(X^{\prime}\right), Y^{\prime}\right\rangle-\langle A(X), Y\rangle-\left\langle A\left(X^{\prime}\right), \tau Y\right\rangle-\left\langle A(\tau X), Y^{\prime}\right\rangle \\
& \quad=\left\langle A\left(X^{\prime}\right), Y^{\prime}\right\rangle-\langle A(X), Y\rangle+\left\langle X^{\prime}, \tau Y-S(Y)\right\rangle-\left\langle\tau X-S(X), Y^{\prime}\right\rangle \\
& \quad=\left\langle A\left(X^{\prime}\right), Y^{\prime}\right\rangle-\langle A(X), Y\rangle+\left\langle S(X), Y^{\prime}\right\rangle-\left\langle S\left(X^{\prime}\right), Y\right\rangle,
\end{aligned}
$$

where we have used the facts that $D^{\prime} \varphi(\alpha(g))=\operatorname{Ad}_{\alpha(g)^{-1}} D \varphi(\alpha(g))$ and $\tau \circ \operatorname{Ad}_{\alpha(g)^{-1}}$ $=\operatorname{Ad}_{\alpha(g)}{ }^{\circ} \tau$. This proves the assertion for $\alpha$. The proof of the other half is similar and details will be left to the reader.

In what follows, we want to show that $P$ can be turned into two distinct Poisson Lie groups. To see this, recall that the maps $\left.\alpha\right|_{U}$ and $\left.\beta\right|_{U}$ are diffeomorphisms $[\mathrm{H}]$. Pushing the group structure on $U$ forward under $\left.\alpha\right|_{U}$ and $\left.\beta\right|_{U}$, we can equip $P$ with multiplication maps $m_{P}, \tilde{m}_{P}: P \times P \rightarrow P$. In this way, $\left.\alpha\right|_{U}$ and $\left.\beta\right|_{U}$ become isomorphisms of Lie groups.

Proposition 3.8. $U$ is a Poisson Lie subgroup of $\left(G_{0},\{\cdot\}_{\text {Skly. }}\right)$.

Proof. According to [STS2], it is enough to verify that $\mathfrak{u}^{\perp} C\left(\mathfrak{g}_{0}\right)_{A}$ is an ideal (recall that $A$ is a classical $r$-matrix by Proposition 3.1). From the definition of $\mathfrak{u}$, we have $\mathfrak{u}^{\perp}=\sum_{\alpha \in \Delta^{+}} \mathbb{R} \cdot Z_{\alpha}$. If $a \in \mathfrak{g}_{0}, X \in \mathfrak{u}^{\perp}$, we find

$$
\begin{aligned}
{[a, X]_{A} } & =\frac{1}{2}\left(\left[a_{-}-a_{+}, X\right]-[a, X]\right) \\
& =-\left[\frac{1}{2} a_{0}+a_{+}, X\right]
\end{aligned}
$$

from which it follows that $[a, X]_{A} \in \mathfrak{u}^{\perp}$, as required.

The induced structure on $U$ will be denoted by $\{,\}_{U}$. Combining Theorem 3.6 with Proposition 3.8 (we do not need the full force of Proposition 3.8, only the fact that $U$ is a Poisson submanifold of $G_{0}$ ), we now have

Corollary 3.9. $\left.\alpha\right|_{U},\left.\beta\right|_{U}:\left(U,\{,\}_{U}\right) \rightarrow\left(P,\{,\}_{P}\right)$ are Poisson diffeomorphisms.

Theorem 3.10. (a) $\left(P, m_{P},\{,\}_{P}\right)$ and $\left(P, \tilde{m}_{P},\{,\}_{P}\right)$ are Poisson Lie groups and the maps $\left.\alpha\right|_{U},\left.\beta\right|_{U}$ are isomorphisms of Poisson Lie groups.

(b) The Bäcklund-Darboux transformation $B:\left(P, m_{P},\{,\}_{P}\right) \rightarrow\left(P, \tilde{m}_{P},\{,\}_{P}\right)$ : $\alpha(u) \mapsto \beta(u), u \in U$, is an isomorphism of Poisson Lie groups. 
Proof. (a) The assertion follows from the following commutative diagrams:
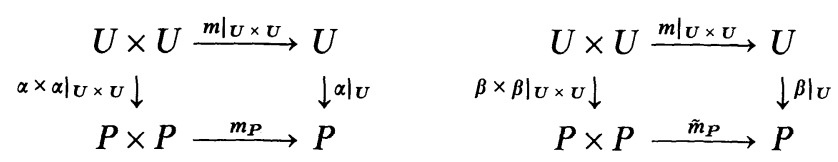

(b) This is clear from the relation $B=\left(\left.\beta\right|_{U}\right) \cdot\left(\left.\alpha\right|_{U}\right)^{-1}$.

Remark 3.11. Let $\mathfrak{g}=s l(l+1, \mathbb{C}), l \geqq 1$ and let $\mathfrak{h}$ be the abelian subalgebra of $\mathfrak{g}$ consisting of diagonal matrices. Then $\mathfrak{h}$ is a Cartan subalgebra, and the CartanKilling form on $\mathfrak{g}$ is given by $\langle X, Y\rangle=2(l+1) \operatorname{tr} X Y$. Let $\lambda_{1}, \ldots, \lambda_{l+1} \in \mathfrak{h}^{*}$ be defined by $\lambda_{j}: \operatorname{diag}\left(a_{1}, \ldots, a_{l+1}\right) \mapsto a_{j}$ and let $\alpha_{i}=\lambda_{i}-\lambda_{i+1}, 1 \leqq i \leqq l$. The set $S=\left\{\alpha_{1}, \ldots, \alpha_{l}\right\}$ is a simple system of roots and the corresponding positive system is $\Delta^{+}=\left\{\lambda_{i}-\lambda_{j} \mid 1\right.$ $\leqq i<j \leqq l+1\}$. For this particular choice of the Cartan subalgebra and the positive system, $\tau$ is the map $X \mapsto-X^{*}, \mathfrak{g}_{0}=s l(l+1, \mathbb{R}), \mathfrak{f}=\operatorname{so}(l+1, \mathbb{R})$ and $\mathfrak{u}$ is the subalgebra of $\mathfrak{g}_{0}$ consisting of upper triangular matrices. We have $G_{0}=S L(l+1, \mathbb{R})$, and the maps $\alpha$ and $\beta$ are given by $\alpha(g)=g^{T} g, \beta(g)=g g^{T}$. It is not hard to check that the subset of "bidiagonals"

$$
\mathscr{B}=\left\{L(a, b)=\left(\begin{array}{cccc}
a_{1} & b_{1} & 0 & \\
& \ddots & \ddots & \\
0 & & \ddots & b_{l} \\
& & & a_{l+1}
\end{array}\right) \in G_{0} a_{i}>0,1 \leqq i \leqq l+1, b_{j}>0,1 \leqq j \leqq l\right\}
$$

is a symplectic leaf of $\left(G_{0},\{\}_{\text {Skly }}\right)$ (see [DDLT]). Thus, it follows from Theorem 3.6 that the maps $L(a, b) \mapsto \alpha(L(a, b)), L(a, b) \mapsto \beta(L(a, b))$ are Poisson maps. This special case was first obtained by Flaschka who computed the Poisson brackets of the various coordinate functions $[\mathrm{F}]$.

Now, although we present the above results within the framework of real, semisimple Lie groups, it is clear that the computations should go through for a reductive group like $G L(n, \mathbb{R})$. Indeed, we have the Iwasawa decomposition $g l(n, \mathbb{R})=\mathfrak{v} \oplus \mathrm{t}$, where $\mathfrak{o}=0(n, \mathbb{R})$ and $\mathrm{t}$ is the subalgebra of upper triangular matrices. Therefore, if $\Pi_{\mathrm{v}}, \Pi_{\mathrm{t}}$ denote the associated projections, then $\Pi_{\mathrm{v}}-\Pi_{\mathrm{t}}$ is a classical $r$-matrix which verifies hypothesis (H) [LP]. Consequently, the identity component $G L^{+}(n, \mathbb{R})$ of $G L(n, \mathbb{R})$, say, is equipped with two Poisson structures as in Proposition 3.3. Moreover, the identity component $T^{+}(n, \mathbb{R})$ of the upper triangular group is a Poisson Lie group isomorphic to the symmetric space of real, $n \times n$ positive definite matrices in two ways. In the rest of the section, we consider the complete integrability of the SVD flow

$$
\dot{u}=\left(\Pi_{\mathrm{o}}\left(u u^{T}\right)\right) u-u\left(\Pi_{\mathrm{o}}\left(u^{T} u\right)\right), \quad u \in T^{+}(n, \mathbb{R})
$$

generated by the Hamiltonian $\operatorname{tr}\left(u^{T} u\right)$. As in Proposition 3.4, if $u$ satisfies the above equation, then the positive definite matrix $\alpha(u)=u^{T} u$ solves the Toda flow

$$
\left(u^{T} u\right)^{\cdot}=\left[\Pi_{\mathrm{o}}\left(u^{T} u\right), u^{T} u\right] .
$$

Now, the Toda flow on positive definite matrices equipped with the induced structure from $\left(G L^{+}(n, \mathbb{R}),\{\},\right)$ can be shown to be completely integrable on generic symplectic leaves using the recursion relations in [LP]. Therefore, the same must hold for the SVD flow on $T^{+}(n, \mathbb{R})$ as the two Hamiltonian systems are isomorphic under $u \mapsto \alpha(u)=u^{T} u$. To describe the integrals, we introduce the 
following notation. For an $n \times n$ matrix $M$, let $(M)_{k}$ be the $(n-k) \times(n-k)$ matrix obtained by deleting the first $k$ rows and the last $k$ columns of $M$ and define

$$
P_{k}(M, \lambda)=\operatorname{det}(M-\lambda)_{k}=\sum_{r=0}^{n-2 k} E_{r k}(M) \lambda^{n-2 k-r}, \quad 0 \leqq k \leqq\left[\frac{n}{2}\right] .
$$

Then the sign of $E_{o k} \circ \alpha, 0 \leqq k \leqq\left[\frac{n}{2}\right]$ is constant on the symplectic leaves of the structure $\{,\}_{T^{+}(n, \mathbb{R})}$ induced from $\left(G L^{+}(n, \mathbb{R}),\{,\}_{\text {Skly. }}\right)$. Therefore, the set

$$
W=\left\{u \in T^{+}(n, \mathbb{R}) \mid E_{o k}\left(u^{T} u\right) \neq 0, k=1, \ldots,\left[\frac{n}{2}\right]\right\}
$$

is foliated by the symplectic leaves of $\{,\}_{T^{+}(n, \mathbb{R})}$. For $u \in W$, define $I_{r k}(u)$ $=E_{r k}\left(u^{T} u\right) / E_{o k}\left(u^{T} u\right), 0 \leqq k \leqq\left[\frac{1}{2}(n-1)\right], 1 \leqq r \leqq n-2 k$.

Theorem 3.12. (a) The symplectic leaves of $\{,\}_{T^{+}(n, \mathbb{R})}$ in $W$ are of dimension $2\left[\frac{n^{2}}{4}\right]$, being the level set of the Casimir functions $I_{n-2 k, k}, 1 \leqq k \leqq\left[\frac{n-1}{2}\right]$.

(b) The SVD flow is completely integrable on the symplectic leaves in W. The $\left[\frac{n^{2}}{4}\right]$ Poisson commuting integrals are given by $I_{r k}, 0 \leqq k \leqq\left[\frac{n-1}{2}\right], 1 \leqq r \leqq n-2 k-1$.

\section{Analog of Miura Maps and Bäcklund-Darboux Transformations for Periodic Toda Flows}

We first introduce the periodic Toda flows and the associated modified equations on some Banach Lie groups contained in $L G L(n, \mathbb{C})$. The paper [GW] provides some of the technicalities for this part.

Let $G$ be $G L(n, \mathbb{C})$. The Lie algebra $\mathfrak{g}_{0}=g l(n, \mathbb{R})$ is a real form of $g=\operatorname{Lie}(G)$ and $G_{0}=G L^{+}(n, \mathbb{R})$ is a corresponding Lie group. Denote by $\sigma$ the involution of $G$ and $\mathfrak{g}$ defined by this real form. Also, denote by $\tau$ the automorphism of $G$ defined by $\tau(g)=\left(g^{-1}\right)^{*}$. The induced map of $\tau$ on $g$ will also be denoted by the same symbol. We have the decomposition $\mathfrak{g}_{0}=\mathfrak{f} \oplus \mathfrak{u}$ (with associated projections $\Pi_{\mathfrak{t}}, \Pi_{\mathfrak{u}}$ ), where $\mathfrak{f}=0(n, \mathbb{R})$ and $\mathfrak{u}$ is the subalgebra of $n \times n$ upper triangular matrices. On the group level, this corresponds to $G_{0}=K U$, where $K=O(n, \mathbb{R})$, and $U$ is the subgroup of upper triangular matrices with positive diagonal entries. Therefore, if we define

$$
R=\Pi_{\mathfrak{f}}-\Pi_{\mathfrak{u}}, \quad A=\frac{1}{2}\left(R-R^{*}\right), \quad \text { and } \quad S=\frac{1}{2}\left(R+R^{*}\right) .
$$

Then $R$ and $A$ are solutions of (mYB) so that $R$ is a classical $r$-matrix which satisfies hypothesis $(\mathrm{H})$ [LP].

Let $\widetilde{G}=C^{\infty}\left(S^{1}, G\right)$ be the smooth loop group with the $C^{\infty}$ topology. $\widetilde{G}$ is a Fréchet Lie group with the Lie algebra $\tilde{\mathfrak{g}}=C^{\infty}\left(S^{1}, \mathfrak{g}\right)$. We extend the conjugation $\sigma$ of $G$ and $\mathfrak{g}$ to a conjugation on the loop group and algebra by setting $(\sigma f)(z)$ $=\sigma(f(\bar{z})),|z|=1$. Similarly, we extend $\tau$ to $\widetilde{G}$ and $\tilde{\mathfrak{g}}$ by $(\tau f)(z)=\tau(f(z)),|z|=1$. 
Notation 4.1. For $g \in \widetilde{G}$, we use the simpler notation $g^{*}$ to stand for $\tau\left(g^{-1}\right)$.

Let $\widetilde{G}_{0}$ and $\tilde{\mathrm{g}}_{0}$ be the fixed point sets of the extended $\sigma$. We shall use the following nondegenerate ad-invariant pairing on $\tilde{\mathfrak{g}}_{0}$ :

$$
\langle X, Y\rangle=\oint_{|z|=1} \operatorname{tr}(X(z) Y(z)) \frac{d z}{z}, \quad d z=\frac{d z}{2 \pi i} .
$$

Obviously, $\langle\tau X, \tau Y\rangle=\langle X, Y\rangle$.

Following $[\mathrm{GW}]$, choose a symmetric weight function $w: \mathbb{Z} \rightarrow \mathbb{R}_{+}$which is rapidly increasing in the sense that

$$
\lim _{h \rightarrow \infty} w(n) n^{-s}=\infty, \quad \forall s>0 .
$$

Also, assume that $w$ is of non-analytic type:

$$
\lim _{h \rightarrow \infty} w(n)^{1 / n}=1
$$

Let $X \in \tilde{\mathfrak{g}}_{0}$ be given by $X(z)=\sum_{-\infty}^{\infty} X_{n} z^{n},|z|=1$. We define $\|X\|_{w}=\sum_{-\infty}^{\infty}\left\|X_{n}\right\| w(n)$, where $\|\cdot\|$ is a norm on $\mathfrak{g}_{0}$. Also, set $\left(P_{+} X\right)(z)=\sum_{n>0} X_{n} z^{n}$ and $\left(P_{-} X\right)(z)=\sum_{n<0}^{-\infty} X_{n} z^{n}$. Consider the Banach Lie group

$$
\widetilde{G}_{0 w}=\left\{g \in \widetilde{G}_{0} \mid\|g\|_{w}<\infty\right\}
$$

with Lie algebra

$$
\tilde{\mathfrak{g}}_{0 w}=\left\{X \in \tilde{\mathfrak{g}}_{0} \mid\|X\|_{w}<\infty\right\} .
$$

We have the decompositions

$$
\tilde{\mathfrak{g}}_{0 w}=\tilde{\mathfrak{I}}_{w} \oplus \tilde{\mathfrak{u}}_{w}=\tilde{\mathfrak{I}}_{w} \oplus \tilde{\mathfrak{p}}_{w},
$$

where $\tilde{\mathfrak{f}}_{w}, \tilde{\mathfrak{p}}_{w}$ are the +1 and -1 eigenspaces of $\tau \mid \tilde{\mathfrak{g}}_{0 w}$ and

$$
\tilde{\mathfrak{u}}_{w}=\left\{X \in \tilde{\mathfrak{g}}_{0 w} \mid P_{-} X=0, X_{0} \in \mathfrak{u}\right\} .
$$

On the group level, this corresponds to the Iwasawa decomposition and the polar decomposition of $\widetilde{G}_{0 w}[\mathrm{GW}]$ :

$$
\tilde{G}_{0 w}=\tilde{K}_{w} \cdot \tilde{U}_{w}, \quad \tilde{G}_{0 w}=\tilde{K}_{w} \cdot \tilde{P}_{w},
$$

where

$$
\begin{aligned}
& \widetilde{K}_{w}=\left\{g \in \widetilde{G}_{0 w} \mid g^{*} g=I_{n}\right\}, \quad \widetilde{U}_{w}=\left\{g \in \widetilde{G}_{0 w} \mid P_{-} g=0, g_{0} \in U\right\} \quad \text { and } \\
& \tilde{P}_{w}=\exp \left(\tilde{\mathfrak{p}}_{w}\right) \text {. }
\end{aligned}
$$

Denote by $\Pi_{\tilde{\mathfrak{f}}_{w}}, \Pi_{\tilde{\mathfrak{u}}_{w}}$ the projection operators relative to the splitting $\tilde{\mathfrak{g}}_{0 w}=\tilde{\mathfrak{f}}_{w} \oplus \tilde{\mathfrak{u}}_{w}$. We define

$$
R^{\#}=\Pi_{\tilde{\mathfrak{f}}_{w}}-\Pi_{\tilde{\mathfrak{u}}_{w}}, \quad A^{\#}=\frac{1}{2}\left(R^{\#}-\left(R^{\#}\right)^{*}\right), \quad S^{\#}=\frac{1}{2}\left(R^{\#}+\left(R^{\#}\right)^{*}\right) .
$$

Proposition 4.2. $R^{\#} \in$ End $\tilde{\mathfrak{g}}_{0 w}$ is a solution of (mYB) which satisfies hypothesis $(\mathrm{H})$. Proof. Since $\tilde{\mathfrak{f}}_{w}$ and $\tilde{\mathfrak{u}}_{w}$ are subalgebras of $\tilde{\mathfrak{g}}_{0 w}$, it follows from [STS1] that $R^{\#}$ is a solution of (mYB). Now, $R^{\#} X=P_{-} X+R X_{0}-P_{+} X+2 \tau P_{-} X$. On the other hand, 
we find $\left(R^{\#}\right)^{*} X=-P_{-} X+R^{*} X_{0}+R_{+} X+2 \tau P_{-} X$. Therefore, $A^{\#} X=P_{-} X$ $+A X_{0}-P_{+} X$. To show $A^{\#}$ is a solution of (mYB), observe that

$$
\left(1 \mp A^{\#}\right)\left[\left(1 \pm A^{\#}\right) X,\left(1 \pm A^{\#}\right) Y\right]=8 A_{\mp}\left[A_{ \pm} X_{0}, A_{ \pm} Y_{0}\right],
$$

where $A_{ \pm}=\frac{1}{2}(A \pm 1)$. Adding the two expressions gives

$$
\begin{aligned}
& 2[X, Y]+2\left[A^{\#} X, A^{\#} Y\right]-4 A^{\#}[X, Y]_{A^{*}} \\
& \quad=2\left[X_{0}, Y_{0}\right]+2\left[A X_{0}, A Y_{0}\right]-4 A\left[X_{0}, Y_{0}\right]_{A} \\
& \quad=0
\end{aligned}
$$

as required.

Definition 4.3. (a) A periodic Toda flow on $\widetilde{G}_{0 w}$ is defined by an equation of the form

$$
\dot{g}=\frac{1}{2} R^{\#}(D \tilde{\varphi}(g)) g-\frac{1}{2} g R^{\#}(D \tilde{\varphi}(g)), \quad g \in \widetilde{G}_{0 w},
$$

where $\tilde{\varphi}(g)=\oint_{|z|=1} \varphi(g(z)) \frac{d z}{z}, \varphi \in C^{\infty}(G)$ being a central function.

(b) The modified periodic Toda equation on $\widetilde{G}_{0 w}$ corresponding to a central function $\varphi \in C^{\infty}(G)$ is the equation

$$
\dot{g}=\frac{1}{2} A^{\#}\left(D \tilde{h}_{\varphi}(g)\right) g-\frac{1}{2} g A^{\#}\left(D^{\prime} \tilde{h}_{\varphi}(g)\right), \quad g \in \widetilde{G}_{0 w},
$$

where $\tilde{h}_{\varphi}(g)=\oint_{|z|=1} \varphi\left(g^{*}(z) g(z)\right) \frac{d z}{z}$.

From Proposition 4.2, it now follows from [LP] that (4.12) and (4.13) are Hamiltonian systems.

Proposition 4.4. (a) Equation (4.12) is the Hamiltonian system in the Poisson structure

$$
\begin{aligned}
\left\{\varphi_{1}, \varphi_{2}\right\}=\frac{1}{2}\left\langle A^{\#}\left(D^{\prime} \varphi_{1}\right), D^{\prime} \varphi_{2}\right\rangle & -\frac{1}{2}\left\langle A^{\#}\left(D \varphi_{1}\right), D \varphi_{2}\right\rangle+\frac{1}{2}\left\langle S^{\#}\left(D \varphi_{1}\right), D^{\prime} \varphi_{2}\right\rangle \\
& -\frac{1}{2}\left\langle S^{\#}\left(D^{\prime} \varphi_{1}\right), D \varphi_{2}\right\rangle, \quad \varphi_{1}, \varphi_{2} \in C^{\infty}\left(\widetilde{G}_{0 w}\right)
\end{aligned}
$$

corresponding to $\tilde{\varphi}$.

(b) Equation (4.13) is the Hamilton's equation generated by $\tilde{h}_{\varphi}$ in the Sklyanin structure

$$
\begin{gathered}
\left\{\varphi_{1}, \varphi_{2}\right\}_{\text {Skly. }}=\frac{1}{2}\left\langle A^{\#}\left(D^{\prime} \varphi_{1}\right), D^{\prime} \varphi_{2}\right\rangle-\frac{1}{2}\left\langle A^{\#}\left(D \varphi_{1}\right), D \varphi_{2}\right\rangle, \\
\varphi_{1}, \varphi_{2} \in C^{\infty}\left(\widetilde{G}_{0 w}\right) .
\end{gathered}
$$

For the Hamiltonians $\tilde{\varphi}$ and $\tilde{h}_{\varphi}$, we have

$$
\begin{aligned}
D \tilde{\varphi}(g)(z) & =D^{\prime} \tilde{\varphi}(g)(z)=D^{\prime} \varphi(g(z)), \\
D \tilde{h}_{\varphi}(g)(z) & =2 D \varphi\left(g(z) g^{*}(z)\right)
\end{aligned}
$$

and

$$
D^{\prime} \tilde{h}_{\varphi}(g)(z)=2 D \varphi\left(g^{*}(z) g(z)\right), \quad|z|=1 .
$$

In particular, $D \tilde{h}_{\varphi}(g), D^{\prime} \tilde{h}_{\varphi}(g) \in \tilde{\mathfrak{p}}_{w}$. As $A^{\#}(X)=\pi_{\tilde{\mathfrak{f}}_{w}} X$ for $X \in \tilde{\mathfrak{p}}_{w}$, a calculation similar to the one in Proposition 3.4 shows that we have the following result. 
Proposition 4.5. If $g \in \widetilde{G}_{0 w}$ solves the modified periodic Toda equation (4.13), then both $g^{*} g$ and $g^{*}$ are solutions of the periodic Toda equation(4.12).

Now, for $g \in \widetilde{G}_{0 w}$, it follows from [GW] that $g g^{*}, g^{*} g \in \widetilde{P}_{w}$. Therefore, we have maps $\tilde{\alpha}, \widetilde{\beta}: \widetilde{G}_{0 w} \rightarrow \widetilde{P}_{w}$ given by $\tilde{\alpha}(g)=g^{*} g, \widetilde{\beta}(g)=g^{*} g$.

Proposition 4.6. (a) $\widetilde{P}_{w}$ is a Poisson submanifold of $\left(\widetilde{G}_{0 w},\{\cdot\}\right)$.

(b) $\widetilde{U}_{w}$ is a Poisson subgroup of $\left(\widetilde{G}_{0 w},\{\cdot\}_{\text {Skly. }}\right)$.

Proof. (a) With $\mathfrak{f}$ replaced by $\tilde{\mathfrak{I}}_{w}, \mathfrak{u}$ by $\tilde{\mathfrak{u}}_{w}$ and so on in the proof of Proposition 3.5, everything goes through just the same as before.

(b) We have to show $\tilde{\mathfrak{u}}_{w}^{\perp} \subset\left(\tilde{\mathfrak{g}}_{0 w}\right)_{A^{*}}$ is an ideal. Now,

$$
\tilde{\mathfrak{u}}_{w}^{\perp}=\left\{X \in \tilde{\mathfrak{g}}_{0 w} \mid P_{-} X=0, X_{0} \in \mathfrak{u}^{\perp}\right\},
$$

where $\mathfrak{u}^{\perp}$ consists of real, $n \times n$ strictly upper triangular matrices.

For $X \in \tilde{\mathfrak{g}}_{0 w}, Y \in \tilde{\mathfrak{u}}_{w}^{\perp}$, we have

$$
[X, Y]_{A^{\#}}=\left[A_{-} X_{0}-P_{+} X, P_{+} Y+Y_{0}\right], \quad A_{-}=\frac{1}{2}(A-1)
$$

and clearly, $P_{-}[X, Y]_{A^{*}}=0$. As the constant term in $[X, Y]_{A^{*}}$ is given by $\left[A_{-} X_{0}, Y_{0}\right] \in \mathfrak{u}^{\perp}$, we conclude that $[X, Y]_{A_{\#}} \in \tilde{\mathfrak{u}}_{w}^{\perp}$.

Notation 4.7. The induced Poisson structures on $\widetilde{P}_{w}$ and $\widetilde{U}_{w}$ will be denoted by $\{,\}_{\tilde{P}_{w}}$ and $\{,\}_{\tilde{U}_{w}}$ respectively.

Theorem 4.8. $\tilde{\alpha}, \widetilde{\beta}:\left(\widetilde{G}_{0 w},\{\cdot\}_{\text {Skly. }}\right) \rightarrow\left(\widetilde{P}_{w},\{\cdot\}_{\tilde{P}_{w}}\right)$ are Poisson maps.

The proof of this theorem proceeds as in Theorem 3.6 because the properties of $A^{\#}$ and $S^{\#}$ are analogous to those of $A$ and $S$ in Lemma 3.7. Indeed, we have

Proposition 4.9. For all $X \in \tilde{\mathfrak{g}}_{0 w}$,

(a) $\tau\left(A^{\#} X\right)=-A^{\#}(\tau X)$,

(b) $A^{\#} X+S^{\#}(\tau X)=X$.

Proof. (a) For $X \in \tilde{\mathfrak{g}}_{0 w}$, we can check that $P_{-}(\tau X)=\tau\left(P_{+} X\right),(\tau X)_{0}=\tau X_{0}$ and $A\left(\tau X_{0}\right)=-\tau\left(A X_{0}\right)$. Therefore,

$$
\begin{aligned}
A^{\#}(\tau X) & =\tau\left(P_{+} X\right)-\tau\left(A X_{0}\right)-\tau\left(P_{-} X\right) \\
& =-\tau\left(A^{\#} X\right) .
\end{aligned}
$$

(b) The assertion follows from $S^{\#}(\tau X)=S\left(\tau X_{0}\right)+2 P_{+} X$ and the property $A X_{0}+S\left(\tau X_{0}\right)=X_{0}$.

Now, we are ready to state the analog of Theorem 3.9. But first, note that $\tilde{\alpha}_{\tilde{U}_{w}}$, $\left.\widetilde{\beta}\right|_{\tilde{U}_{w}}$ are diffeomorphisms. To see this, suppose $u^{*} u=u^{\prime *} u^{\prime}, u, u^{\prime} \in \widetilde{U}_{w}$. Then by Liouville's theorem, $\left(u^{*}\right)^{-1} u^{*}=u^{\prime} u^{-1}=\delta$, where $\delta$ is a diagonal matrix with positive diagonal entries. Thus, it follows from $u^{*}=u^{*} \delta=u^{\prime *} \delta^{-1}$ that $\delta^{2}=1$. Consequently, $\delta=1$ and we conclude that $\tilde{\alpha}_{\tilde{U}_{w}}$ is injective. Now, let $\exp (X) \in \widetilde{P}_{w}$, with $X \in \tilde{\mathfrak{p}}_{w}$. Since $\widetilde{G}_{0 w}=\widetilde{K}_{w} \cdot \widetilde{U}_{w}$, there exists $k \in \tilde{K}_{w}, u \in \widetilde{U}_{w}$ such that $\exp \left(\frac{X}{2}\right)=k u$. Hence, $\exp (X)=u^{*} u$ and so $\left.\tilde{\alpha}\right|_{\tilde{U}_{w}}$ is surjective. In a similar way, one proves $\left.\widetilde{\beta}\right|_{\tilde{U}_{w}}$ is a diffeomorphism. Since $\tilde{U}_{w}$ is a Lie group, we can equip $\widetilde{P}_{w}$ with multiplication maps $m_{\tilde{P}_{w}}, \tilde{m}_{\tilde{P}_{w}}$ so that $\left.\tilde{\alpha}\right|_{\tilde{U}_{w}},\left.\widetilde{\beta}\right|_{\tilde{U}_{w}}$ become isomorphisms of Lie groups as before. 
Theorem 4.10. (a) $\left(\tilde{P}_{w}, m_{\tilde{P}_{w} w},\{,\}_{\tilde{P}_{w}}\right)$ and $\left(\tilde{P}_{w}, \tilde{m}_{\tilde{P}_{w}},\{,\}_{\tilde{P}_{w}}\right)$ are Poisson Lie groups and the diffeomorphisms $\left.\tilde{\alpha}\right|_{\tilde{U}_{w}},\left.\widetilde{\beta}\right|_{\tilde{U}_{w}}$ are isomorphisms of Poisson Lie groups.

(b) The Bäcklund-Darboux transformation $\widetilde{B}:\left(\widetilde{P}_{w}, \quad m_{\tilde{P}_{w}},\{,\} \tilde{P}_{w}\right) \rightarrow\left(\tilde{P}_{w}, \tilde{m}_{\tilde{P}_{w}}\right.$, $\left.\{,\}_{\tilde{P}_{w}}\right): \tilde{\alpha}(u) \mapsto \widetilde{\beta}(u), u \in \widetilde{U}_{w}$, is an isomorphism of Poisson Lie groups.

We can apply Proposition 4.6, Theorem 4.8 and Theorem 4.10 to the periodic Toda lattice. Consider loops in $\widetilde{G}_{0 w}$ of the form

$$
u_{a, b}(z)=\left(\begin{array}{cccc}
a_{1} & b_{1} & & 0 \\
& \ddots & \ddots & \\
& & \ddots & b_{n-1} \\
0 & & & a_{n}
\end{array}\right)+\left(\begin{array}{ccc}
0 & & 0 \\
\vdots & \ddots & \\
b_{n} & \ldots & 0
\end{array}\right) z, \quad|z|=1 .
$$

It is straightforward to check that loops of such a form constitute a Poisson submanifold of $\left(\widetilde{G}_{0 w},\{,\}_{\text {Skly. }}\right)$. Therefore, the modified periodic Toda flow

$$
\dot{u}_{a, b}=u_{a, b} A^{\#}\left(u_{a, b}^{*} u_{a, b}\right)-A^{\#}\left(u_{a, b} u_{a, b}^{*}\right) u_{a, b}
$$

is Hamiltonian. Indeed, it is equivalent to the $2 n$-periodic Kac-van Moerbeke lattice $\dot{\alpha}_{i}=\alpha_{i}\left(\alpha_{i+1}^{2}-\alpha_{i-1}^{2}\right), \alpha_{i+2 n} \equiv \alpha_{i}$ upon letting $\alpha_{2 i-1}=a_{i}, \alpha_{2 i}=b_{i}, i=1, \ldots, n$. Clearly, $\tilde{\alpha}\left(u_{a, b}\right)$ is an $n$-periodic Toda matrix of the form

$$
\left(\begin{array}{ccc}
0 & \ldots & d_{n} \\
& \ddots & \vdots \\
0 & & 0
\end{array}\right) z^{-1}+\left(\begin{array}{cccc}
c_{1} & d_{1} & & \\
d_{1} & \ddots & \ddots & 0 \\
& \ddots & \ddots & d_{n-1} \\
0 & & d_{n-1} & c_{n}
\end{array}\right)+\left(\begin{array}{ccc}
0 & & 0 \\
\vdots & \ddots & \\
d_{n} & \ldots & 0
\end{array}\right) z .
$$

Moreover, if $u_{a, b}$ satisfies the modified equation above, then $\left(\tilde{\alpha}\left(u_{a, b}\right)\right)^{*}=\left[\tilde{\alpha}\left(u_{a, b}\right)\right.$, $\left.\Pi_{\tilde{f}_{w}} \tilde{\alpha}\left(u_{a, b}\right)\right]$ which is the equation of the periodic Toda lattice. Of course, similar remarks apply to $\widetilde{\beta}\left(u_{a, b}\right)$. Now, it is elementary to check that

$$
\widetilde{T}=\left\{\tilde{\alpha}\left(u_{a, b}\right) \mid u_{a, b} \in \widetilde{G}_{0 w} \text { is of the form in (4.14) }\right\}
$$

is a Poisson submanifold of $\left(\widetilde{P}_{w},\{,\} \tilde{P}_{w}\right)$. Furthermore, if $\tilde{\alpha}\left(u_{a, b}\right) \in \widetilde{T}$, then also $\widetilde{\beta}\left(u_{a, b}\right) \in \widetilde{T}$ and vice versa. Thus, the maps $u_{a, b} \mapsto \tilde{\alpha}\left(u_{a, b}\right), u_{a, b} \mapsto \widetilde{\beta}\left(u_{a, b}\right)$ and $\tilde{\alpha}\left(u_{a, b}\right) \mapsto \widetilde{\beta}\left(u_{a, b}\right)$ are Poisson maps when the domains and ranges are equipped with the appropriate induced structures. We leave it to the reader to verify that similar relations are also true for the nonperiodic version of the Toda lattice.

Acknowledgements. The work of the first author was supported in part by NSF Grant DMS-9001857. The authors would like to thank $H$. Flaschka who showed them the map $B \mapsto B^{T} B$ from the bidiagonal matrices to the tridiagonal matrices is a Poisson map.

\section{References}

[A] Adler, M.: On the Bäcklund transformation for the Gelfand-Dickey equations. Commun. Math. Phys. 80, 517-527 (1981)

[C] Chu, M.: A differential equation approach to the singular value decomposition of bidiagonal matrices. Lin. Alg. Appl. 80, 71-80 (1986)

[D] Deift, P.: Applications of a commutation formula. Duke Math. J. 45, 267-310 (1978)

[DDLT] Deift, P., Demmel, J., Li, L.-C., Tomei, C.: The bidiagonal singular value decomposition and Hamiltonian mechanics. SIAM. J. Numer. Anal. 28, 1463-1516 (1991) 
[Drin] Drinfeld, V.: Hamiltonian structures on Lie groups, Lie bialgebras and the geometric meaning of the classical Yang-Baxter equations. Sov. Math. Dokl. 27, 68-71 (1983)

[F] Flaschka, H.: Private communication

[GD1] Gelfand, I., Dickey, L.: Fractional power of operators and Hamiltonian systems. Funct. Anal. Appl. 10, 259-273 (1976)

[GD2] Gelfand, I., Dickey, L.: A family of Hamiltonian structures related to nonlinear integrable differential equations. Preprint no. 136, Inst. Appl. Math. USSR Acad. Sci. 1978 (in Russian), English transl. in Collected papers of I. M. Gelfand, Vol. 1. Berlin, Heidelberg, New York: Springer 1987, pp. 625-646

[GW] Goodman, R., Wallach, N.: Structure and unitary cocycle representations of loop groups and the group of diffeomorphisms of the circle. J. Reine Angew. Math. 347, 69-133 (1984)

[H] Helgason, S.: Differential geometry, Lie groups, and symmetric spaces. New York: Academic Press 1978

[KW] Kupershmidt, B., Wilson, G.: Modifying Lax equations and second Hamiltonian structure. Invent. Math. 62, 403-436 (1981)

[KvM] Kac, M., van Moerbeke, P.: On some periodic Toda lattices. Proc. Natl. Acad. Sci. USA 72, 1627-1629 (1975)

[LP] Li, L.-C., Parmentier, S.: A new class of quadratic Poisson structures and the YangBaxter equation. C. R. Acad. Sci., Paris Sér. I Math. 307, 279-281 (1988). Nonlinear Poisson structures and $r$-matrices. Commun. Math. Phys. 125, 545-563 (1989)

[M] Miura, R.: Korteweg-de Vries equation and generalizations. I. A remarkable explicit nonlinear transformation. J. Math. Phys. 9, 1202-1204 (1968)

[STS1] Semenov-Tian-Shansky, M.: What is a classical $r$-matrix?. Funct. Anal. Appl. 17, 259-272 (1983)

[STS2] Semenov-Tian-Shansky, M.: Dressing transformations and Poisson group actions. Publ. RIMS, Kyoto Univ. 21, 1237-1260 (1985)

[V] Varadarajan, V.S.: Lie groups, Lie algebras, and their representations. Berlin, Heidelberg, New York: Springer 1984

[WE] Wahlquist, H., Estabrook, F.: Bäcklund transformation for solutions of the Kortewegde Vries equation. Phys. Rev. Lett. 31, 1386-1390 (1973)

Communicated by B. Simon 